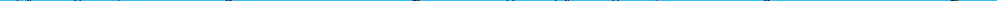

\title{
La evolución de
} la evaluación: por el

\section{mejoramiento del ejercicio} docente ${ }^{1}$

Por Fabiola Inés Hernández Barriga², Carlos Alfonso Orozco ${ }^{3}$, Lina María Fonseca Ortiz ${ }^{4}$, Iliana Fernández Peña ${ }^{5}$, Lida Rubiela Fonseca Gómez ${ }^{6}$, Esther Gutiérrez $\mathrm{Mora}^{7}$

$1 \quad$ Este trabajo es resultado del proyecto de investigación Metaevaluación del proceso de evaluación docente institucional USTA 2012-2014, liderado por la Unidad de Desarrollo Curricular y Formación Docente (UDCFD), Sede Principal Bogotá.

2 Doctora en Gerencia y Política Educativa de la Universidad de Baja California Tepic, México. Magíster en Educación de la Universidad Santo Tomás. Especialista en Educación Personalizada de la Universidad Católica de Manizales. Licenciada en Ciencias de la Educación con Estudios Mayores en Biología de la Universidad Pedagógica Nacional. Correo electrónico: fabiolahernandez@usantotomas.edu.co

3 Magíster en E-learning de la Universidad Autónoma de Bucaramanga, Universitat Oberta de Catalunya. Especialista en Informática y Telemática de la Fundación Universitaria del Área Andina. Ingeniero de Sistemas de la Universidad Nacional Abierta y a Distancia.

$4 \quad$ Magíster en Psicología Jurídica de la Universidad Santo Tomás. Psicóloga de la Konrad Lorenz Fundación Universitaria.

$5 \quad$ Magíster en Ciencias Sociales y Axiología de la Universidad de Holguín “Oscar Lucero Moya”. Licenciada en Educación, especialidad Español - Literatura del Instituto Superior Pedagógico "José de la Luz y Caballero", actualmente, Universidad de Holguín "Oscar Lucero Moya”.

6 Doctora en Educación de la Universidad Pedagógica Nacional. Magíster en Educación de la Uniminuto - Tecnológico de Monterrey. Magíster en Tecnologías Digitales Aplicadas a la Educación de la Universidad Manuela Beltrán. Especialista en Diseño de Ambientes de Aprendizaje de la Uniminuto. Estadística de la Universidad Nacional de Colombia. Docente investigadora de la Facultad de Estadística.

7 Estudiante del Doctorado en Ciencias de la Educación de 
$\mathbf{E}^{\mathrm{n}}$ el 2005, la Universidad Santo Tomás (USTA) comenzó un ejercicio de reflexión acerca de la evaluación docente, la importancia de los procesos asociados y su respectivo impacto sobre la comunidad académica, en virtud de ello, el objetivo versó sobre la realización de una revisión y ajuste del instrumento de evaluación que se aplicaba. Por lo anterior, se entendió la importancia de ser críticos, de aprender de lo vivido, realizar un ejercicio valorativo que permita tomar decisiones de mejoramiento (USTA, 2010).

Con base en la revisión y ajuste del instrumento, en el año 2012 nace, dentro del marco de los proyectos de investigación FoDEIN, una alianza estratégico-investigativa entre la Facultad de Estadística y la Unidad de Desarrollo Curricular y Formación Docente, Sede Principal Bogotá, instancias que conjuntamente pusieron en marcha la investigación denominada "Metaevaluación del proceso de evaluación docente institucional USTA 2012-2014”, cuyo objetivo principal era "generar nuevas y mejores formas de abordar el proceso de evaluación al interior de la Universidad”. En esta primera etapa, se logra entender la potencialidad de la evaluación no sólo como dispositivo de medición de técnicas y variables, sino también comprender otros métodos de orden cualitativo y cotidiano que subyacen a los procesos de enseñanza y aprendizaje. Así, una de las primeras reflexiones que queda en el registro de esta alianza estratégica plantea que,

La evaluación constituye una parte fundamental del proceso pedagógico (enseñanza $y$ aprendizaje), es transversal al currículo $y$ lo nutre en todas sus etapas. De ahí que no sólo permita evidenciar resultados entorno

la Universidad Nacional de la Plata. Magíster en Investigación Social Interdisciplinaria de la Universidad Distrital Francisco José de Caldas. Socióloga de la Universidad Santo Tomás. al desempeño docente, sino también reconocer la eficacia de los métodos pedagógicos, la organización administrativa y física, la adecuada utilización de la tecnología, entre otros, que impactan en el proceso pedagógico. (Gutiérrez y Zambrano, 2017, p. 102)

De otro lado, para la investigación en la USTA resulta importante conocer otros ejercicios y buenas prácticas alrededor de los procesos de evaluación docente, que se dan en otras Instituciones de Educación Superior, por ello, y tal como lo mencionan Montoya, Arbesú, Contreras y Conzuelo (2014), en un estudio realizado en varias universidades de México, Chile y Colombia, la evaluación debe incluir:

1. El uso de diversos instrumentos y variadas fuentes de información.

2. El uso de métodos cualitativos.

3. La relación con el proceso de formación docente y desarrollo profesional de los profesores.

4. La información en un sistema rápido, de fácil acceso y lectura para los propios profesores.

Estos cuatro elementos mencionados se evidencian el proceso riguroso que se da en la USTA, pues, la apuesta investigativa se desarrolló en dos frentes principales: cuantitativo, desde el enfoque del análisis factorial, con el objetivo de medir la validez y confiabilidad del instrumento de evaluación docente; y cualitativo, al abordar y analizar las dimensiones de contexto de la evaluación. Como un valor agregado y a partir del análisis factorial, se realizó una correlación que permitió afirmar que no existe una relación entre la nota definitiva que obtiene el estudiante y la nota que este le otorga al profesor en la evaluación docente, es decir, coloquialmente hablando "no existe un gana-gana, o hagámonos pasito" en la evaluación docente. 
De igual forma, dentro de los grandes hitos que se han logrado con la implementación de este proyecto y asociados a los cuatro elementos mencionados, se encuentran:

- Mejoramiento del aplicativo de evaluación docente de pregrado, en tanto se cuenta con un nuevo sistema ágil y moderno a partir del primer periodo académico de 2017, con alcance nacional Multicampus, el cual ha sido adoptado por las Seccionales y Sedes y se encuentra en proceso de migración en la Decanatura de Educación Abierta y a Distancia, dada su particularidad con respecto a las modalidades.

- Diseño del Plan de Acompañamiento para fortalecer el desempeño docente en el Aula, el cual favorece de manera significativa los estilos de enseñanza y rasgos actitudinales de los profesores, quienes reportan mayor confianza personal y profesional.

- Presentación de los resultados de la investigación a través de la Ponencia "La metaevaluación docente en la Universidad Santo Tomás: el instrumento de evaluación" en la 9. ${ }^{\text {a }}$ edición de la Conferencia Científica Internacional de la Universidad de Holguín, en abril de 2019.

- Diseño de cursos y jornadas de cualificación acordes a los resultados de la evaluación docente.

Para el año 2019 se realizaron las socializaciones de los resultados con los Decanos de División de la Sede Principal, además, se inició el estudio de nuevos ítems que permitan dar cubrimiento a las particularidades de la modalidad a distancia, con el propósito de generar instrumentos pertinentes para la División de Educación Abierta y a Distancia, y de esta manera favorecer su integración al modelo de evaluación a través del aplicativo integrado que funciona en todas las Seccionales y Sedes.

Por ello, la Vicerrectoría Académica General ha estimado oportuno continuar con el proyecto y lo considera un insumo valioso para el mejoramiento constante del quehacer docente en el ejercicio áulico. Debido a esto, la "Metaevaluación" continuó su proceso investigativo y, durante todos estos años, se ha robustecido en relación con sus análisis cuantitativos y cualitativos.

Consecuentemente, a parte de los reconocimientos que se han venido cosechado con este proyecto, se puede afirmar con total seguridad que la construcción y mejoramiento de la evaluación docente en la USTA, implicó una apuesta muy grande en términos de integralidad y articulación de las dimensiones a evaluar. 
Gracias a los análisis realizados en la metaevaluación docente se han podido llegar a comprender los grandes rasgos del ejercicio evaluativo que se aplica a los profesores de la Universidad, por lo tanto esta investigación realmente ha permitido identificar lo disciplinar, lo pedagógico y la identidad institucional como dimensiones valoradas en la práctica docente, y que bajo la concepción del pensamiento tomista se convierten en ejes esenciales para la planeación y desarrollo de los procesos de enseñanza y aprendizaje.

\footnotetext{
Referencias

Gutiérrez, E., \& Zambrano, A. (2019). La dimensión contextual en la comprensión de los procesos de evaluación: La Universidad y su experiencia en el proceso de metaevaluación del desempeño docente. En F. I. Hernández \& L. M. Fonseca (Eds.), Gestión curricular y desarrollo de competencias en estudiantes y docentes Apuesta por la calidad universitaria (pp. 99-122). Bogotá: Ediciones USTA.

Montoya, J., Arbesú, I., Contreras, G., \& Conzuelo, S. (2014). Evaluación de la docencia universitaria en México, Chile y Colombia: Análisis de experiencias. Revista Iberoamericana de Evaluación Educativa, 7(2. ${ }^{\mathrm{a}}$ ed.), 15-42. Recuperado de http://www. rinace.net/riee/numeros/vol7-num2e/art1.pdf

Universidad Santo Tomás. (2010). Dimensión de la política docente. Bogotá: Ediciones USTA.
} 